Meta

Journal des traducteurs

Translators' Journal

\title{
À la recherche d'une terminochronie
}

\section{Bernt Møller}

Volume 43, numéro 3, septembre 1998

URI : https://id.erudit.org/iderudit/003655ar

DOI : https://doi.org/10.7202/003655ar

Aller au sommaire du numéro

\section{Éditeur(s)}

Les Presses de l'Université de Montréal

ISSN

0026-0452 (imprimé)

1492-1421 (numérique)

Découvrir la revue

Citer cet article

Møller, B. (1998). À la recherche d'une terminochronie. Meta, 43(3), 426-438. https://doi.org/10.7202/003655ar

\section{Résumé de l'article}

L'article s'attaque au déficit diachronique en terminologie. La notion d'évolution est appliquée d'abord au micro-niveau, celui du terme, où il convient de distinguer l'évolution du signifiant et celle du signifié. Deux phénomènes relatifs à l'évolution formelle - l'expansion et la réduction - sont présentés. Au macro-niveau, celui d'une terminologie entière, une définition de l'évolution est proposée à partir d'un modèle conçu pour représenter les ensembles diachroniques des termes constituant une terminologie. Dans cette perspective, les lemmes d'un dictionnaire informatique (éditions 1970 et 1985) sont soumis à des analyses structurales diachroniques. Ces analyses montrent que l'importance des lemmes de l'édition de 1970 qui ne se retrouvent pas dans l'édition de 1985 est réduite et que leur structure est complexe. Les analyses révèlent aussi que les lemmes susceptibles de refléter le phénomène de la réduction sont rarissimes.
Ce document est protégé par la loi sur le droit d'auteur. L'utilisation des services d'Érudit (y compris la reproduction) est assujettie à sa politique d'utilisation que vous pouvez consulter en ligne.

https://apropos.erudit.org/fr/usagers/politique-dutilisation/ 


\title{
ÉTUDES TERMINOLOGIQUES ET LINGUISTIQUES
}

\section{À LA RECHERCHE D'UNE TERMINOCHRONIE}

\begin{abstract}
Résumé
L'article s'attaque au déficit diachronique en terminologie.

La notion d'évolution est appliquée d'abord au micro-niveau, celui du terme, où il convient de distinguer l'évolution du signifiant et celle du signifié. Deux phénomènes relatifs à l'évolution formelle - l'expansion et la réduction - sont présentés.

Au macro-niveau, celui d'une terminologie entière, une définition de l'évolution est proposée à partir d'un modèle conçu pour représenter les ensembles diachroniques des termes constituant une terminologie. Dans cette perspective, les lemmes d'un dictionnaire informatique (éditions 1970 et 1985) sont soumis à des analyses structurales diachroniques. Ces analyses montrent que l'importance des lemmes de l'édition de 1970 qui ne se retrouvent pas dans l'édition de 1985 est réduite et que leur structure est complexe. Les analyses révèlent aussi que les lemmes susceptibles de refléter le phénomène de la réduction sont rarissimes.

\section{Abstract}

Discussions of diachronic approaches in terminology are scarce or not to the point. The present paper stresses the necessity of defining evolution as it relates to the signifiant or to the signifié, to the term or to a terminology.

At the term level two very different phenomena are presented. At the terminology level a simple diachronic model is set up with the aim of defining evolution and depicting quantitative relations between diachronic sets of terms. To complete the discussion, the French nouns in the 1970 and 1985 edition of a data processing dictionary are analysed in order to identify diachronic quantitative and structural differences.
\end{abstract}

\section{INTRODUCTION}

La présente contribution tire son origine d'un projet de recherche qui a pris son point de départ dans le domaine de la formation des mots en langue de spécialité. Je me suis intéressé aux répercussions de l'évolution technologique sur l'évolution des terminologies et ai dû constater que la lexicologie, la terminologie ou même la néologie, toutes disciplines traditionnelles, semblent présenter un déficit diachronique qualitatif ou quantitatif, voire les deux à la fois. Ce fait, qui semble être regretté par certains terminologues ${ }^{1}$, rend nécessaires tout d'abord une discussion de base de ce que l'on doit comprendre par évolution dans un contexte terminologique, ensuite une élaboration d'outils aptes à quantifier cette évolution. Mon but sera ici d'aborder la discussion dans une branche de la terminologie que je nomme terminochronie - l'étude de l'évolution des termes et des terminologies.

\section{1. ÉVOLUTION}

Le mot évolution utilisé en terminologie peut cacher plusieurs sens. Si une terminologie est considérée comme «en pleine évolution» ou «en pleine croissance», cela veut probablement dire que beaucoup de termes nouveaux s'y rapportant ont été attestés plus ou moins récemment ou qu'une certaine structure (suffixe, par exemple) sem- 
ble «fréquente» ou «productive» au sein de cette même terminologie. Par contre, l'«évolution d'un terme» est une expression liée aux changements de forme et/ou de sens de termes individuels faisant partie d'une terminologie, et vise soit le phénomène de réduction, soit celui d'expansion, soit les deux à la fois (les termes réduction et expansion seront présentés respectivement dans les sections 2.2 et 2.1). À la lueur de cette confusion ou de ce manque de précision, il me semble essentiel de présenter et discuter quelques modalités relatives à l'évolution.

Bien que l'évolution d'une terminologie en tant qu'unité soit intimement liée à l'évolution de ses parties constituantes, les termes, on aura avantage à séparer ces deux niveaux lors d'une présentation. Du point de vue du procédé d'analyse, la différence, comme nous le verrons, peut être résumée ainsi : au niveau collectif, celui indiqué pour l'ensemble des termes constituant la terminologie, deux ou plusieurs états aléatoires de la progression temporelle sont décrits sous forme d'une accumulation de données individuelles. Au niveau individuel, ce sont par contre les phases successives d'états différents de l'unité terminologique qui sont exposées.

Dans la première partie de cette contribution, je discuterai des particularités liées à l'évolution des termes individuels - je serai donc au micro-niveau. Il me semble naturel de corréler les deux niveaux de l'évolution, mais l'objectif lui-même sera de discuter les modalités relatives à l'évolution des termes en tant qu'unités. Dans l'autre partie, j'aborderai l'aspect collectif de la terminologie - je serai là au macro-niveau. Je présenterai quelques définitions pertinentes et, en me basant sur la terminologie de l'informatique comme elle se manifeste dans certains dictionnaires d'informatique bilingues, je chercherai d'une part à appliquer ces définitions et, d'autre part, à étudier quelques phénomènes spécifiques s'y rapportant.

\section{2. ÉVOLUTION AU MICRO-NIVEAU - CELUI DU TERME}

À mon avis, le principe de base de toute analyse au micro-niveau stipule que l'évolution du signifiant ne doit pas être confondue avec l'évolution du signifié. Trop souvent dans la littérature, cette distinction entre forme et sens n'est pas faite ou bien n'a pas été entreprise de façon rigoureuse, ce qui risque de prêter à confusion. À titre d'exemple, le manuel de terminologie de Daniel Gouadec, qui a pourtant le mérite de traiter sur plusieurs pages des mécanismes de l'évolution terminologique et de conclure le chapitre de façon pertinente ${ }^{2}$, ne semble pas considérer comme essentiel de toujours séparer l'évolution du signifiant de celle du signifié :

[L'évolution] intéresse les termes pris individuellement avec, pour chacun d'eux, un cycle dans lequel se succèdent (globalement), selon un rythme aléatoire, l'emprunt ou la création, puis la spécialisation progressive, puis l'état d'équilibre des termes spécialisés, puis la dé-spécialisation ou vulgarisation, et enfin l'émergence de désignations nouvelles. (Gouadec 1990 : 23)

Il serait préférable, voire indispensable, de repérer l'évolution exprimée soit par la forme. Mais le défi serait également de les corréler sciemment en vue de saisir les caractéristiques évolutives de la dichotomie. Par exemple, la réduction du type $\mathrm{X}$ d'un signifiant s'accompagne-t-elle d'un glissement de sens - et, dans l'affirmative, dans quelles situations de discours?

Du point de vue évolutif, le couple signifiant-signifié peut théoriquement se présenter en quatre combinaisons : 1) ni le signifiant ni le signifié ne changent, 2) le signifié seul change, 3) le signifiant et le signifié changent, 4) le signifiant seul change. La première combinaison n'est intéressante qu'en tant que base de comparaison; la 
deuxième relève de la sémantique, et comme il est de mon intention de me limiter plus ou moins à étudier l'évolution du terme, exprimée par la forme, cette combinaison ne sera pas commentée davantage. Restent les combinaisons 3) et 4) qui seront discutées dans ce qui suit.

\subsection{Expansion}

La troisième catégorie, 3) le signifiant et le signifié changent, est représentée par les analyses de l'expansion des signifiants (voir p. ex. Portelance 1986, 1989). L'objectif de cette approche est plutôt au niveau de l'ensemble d'une terminologie, mais sa base sera indiquée ici. Ce type d'analyses met à l'étude des phénomènes comme la capacité générative, le potentiel combinatoire et les séries paradigmatiques. Il s'agit entre autres choses de répertorier les manifestations individuelles d'expansion formelle des termes de base. Exemple : disque -> disque souple $->$ disque souple double face $->$ disque souple double face double densité. Souvent, les deux aspects du signe linguistique sont corrélés $\mathrm{du}$ fait que ces développements successifs traduisent une spécialisation croissante $\mathrm{du}$ terme de base. Or, souvent n'est pas toujours, et le signifié ne semble jouer aucun rôle pour ce type d'analyse. Ce qui est certain, c'est que le sens est rarement constant. Il est intéressant de noter que les analyses d'expansion sont plutôt interprétées synchroniquement, bien qu'une approche explicitement diachronique serait pertinente.

\subsection{Réduction}

La quatrième catégorie, 4) le signifiant seul change, correspond aux études de réduction du signifiant. Elle me préoccupe particulièrement et sera examinée en un peu plus de profondeur. À première vue, l'expansion et la réduction sont des phénomènes opposés parallèles. Cela n'est que partiellement correct. Il s'agit dans les deux cas de processus qui, respectivement, prolongent et contractent les termes. Mais avec cette différence fondamentale au niveau individuel que le premier processus concerne, grosso modo, le développement d'une forme dont les sens successifs sont souvent apparentés mais non identiques; le second processus, par contre, est un changement de forme dont le sens correspondant est - idéalement - constant tout au long du processus. Malheureusement, cette distinction n'est pas toujours respectée, comme le montre cet exemple pourtant instructif :

La diachronie est toute relative $[. .$.$] , mais les exemples [\ldots]$ abondent qui montrent l'alternance de l'expansion et du rétrécissement syntagmatique. Ainsi le terme «propagation» [...] La propagation sera ascendante, descendante, vers l'avant, vers l'arrière, etc. L'ajout de déterminants fait gagner en précision ce que l'on perd en maniabilité. Mais à l'usage, les syntagmes rétrécissent, la propagation vers l'arrière devient «propagation régressive» et ensuite «rétro-propagation». (Pavel 1989 : 348)

Les procédés de réduction, comme la formation d'acronymes, de sigles, de motsvalises, de composés par juxtaposition, ont déjà été amplement examinés ailleurs, surtout individuellement. Pourtant, il me semble que l'aspect dynamique a été négligé : l'étude des générations de termes, des raccourcissements successifs des signifiants, des modèles diachroniques de formation des termes ${ }^{3} \ldots$

Comment remédier à ce déficit ? En ce qui concerne le phénomène de réduction, la littérature traitant de la formation des termes nous présente quelques exemples de réductions diachroniques. Ainsi, Goffin (1989: 105) mentionne l'exemple protection contre les rayonnements -> protection radiologique -> radioprotection. Il constitue une chaîne diachronique de signifiants pour un signifié présumé constant. Il serait souhaitable de généraliser l'information contenue dans cette chaîne afin d'avoir une vue 
d'ensemble de la réduction des signifiants de la même structure que protection contre les rayonnements. Une chaîne de symboles $\mathrm{A}->\mathrm{B}->\mathrm{C}$ nous aidera dans cette tâche, où «A» symbolise la première forme que revêt une notion lors de son introduction dans la terminologie, «B» la forme suivante, etc. Si nous admettons dans un premier temps que les classes des mots peuvent servir comme constituants d'un maillon de la chaîne, les matrices terminogéniques s'imposent. Nous aurons ainsi pour l'exemple ci-dessus: NPArtN -> NA -> N. Nous savons maintenant qu'un terme de première génération ayant la structure substantif + préposition + article + substantif peut, à titre de terme de deuxième génération, revêtir la structure substantif + adjectif, et que la structure substantif + adjectif peut se retrouver transformée ultérieurement en substantif seul (terme de troisième génération, in casu $)^{4}$.

Le principe de base qu'illustre une chaîne est donc la représentation des réductions successives de l'étendue du signifiant, repérées en discours d'abord, transformées en matrices par la suite - ou inversement: établissement de matrices théoriques d'abord, quête d'attestations par la suite.

Tôt ou tard, nous nous rendrons compte que les constituants des matrices terminogéniques doivent être affinés de façon à englober non seulement les classes de mots, mais également les manifestations de réduction du nombre de syllabes ou de caractères, de même que la suppression des signes d'intégration comme le trait d'union ou les points séparant souvent les lettres des sigles.

Un grand nombre de questions devraient être élucidées contribuant ainsi à privilégier une analyse dynamique des terminologies. Précisons que l'idée présuppose que l'on arrive à une définition de la constance du signifié, ce qui est, bien entendu, une tâche épineuse. Une problématique non moins fondamentale est celle de la dialectique entre temps et forme. Les chaînes traduiront-elles vraiment une progression temporelle, ou est-ce qu'elles refléteront uniquement une différence formelle ${ }^{5}$ ? En raison de ces facteurs, parmi d'autres, l'interprétation des attestations pourrait être faussée. Les chaînes devraient idéalement neutraliser l'effet de ce type de problèmes dont le remède principal est le choix d'un corpus diachronique fortement homogène.

\section{3. ÉVOLUTION AU MACRO-NIVEAU - CELUI DE LA TERMINOLOGIE}

Comme indiqué précédemment, la notion d'évolution appliquée non au microniveau - celui du terme - mais au macro-niveau - celui de l'ensemble de termes constituant une terminologie - paraît floue. Les terminologues et les néologues ne s'intéressent pas en général à l'aspect collectif - voire à l'approche diachronique d'une ou plusieurs terminologies. Toutefois, la discussion portant sur les caractéristiques des langues de spécialités gagnerait à inclure des considérations sur la nature globale des terminologies, y compris des réflexions sur l'évolution de celles-ci.

S'il est tout indiqué d'aborder le micro-niveau de façon qualitative, une approche quantitative s'impose si l'on désire définir la notion d'évolution et des notions connexes au macro-niveau. Dans ce qui suit, un modèle très simpliste sera présenté en vue d'établir ces définitions et de les quantifier.

Le modèle (figure 1) présuppose qu'un terme soit existe, soit n'existe pas à un moment donné, et que chaque terme a une vie délimitée dans le temps et qui peut être vérifiée en discours.

Le modèle représente la quantité globale des termes constituant une terminologie au moment $\mathrm{t} 1$ et $\mathrm{t} 2$ dans la vie d'une terminologie. Deux moments seulement pour simplifier, ce qui exclut des données situées dans l'intervalle ]t2; $1\left[^{6}\right.$. Nous avons trois ensembles A, B et C. L'ensemble A est constitué de la totalité des signifiants qui sont 


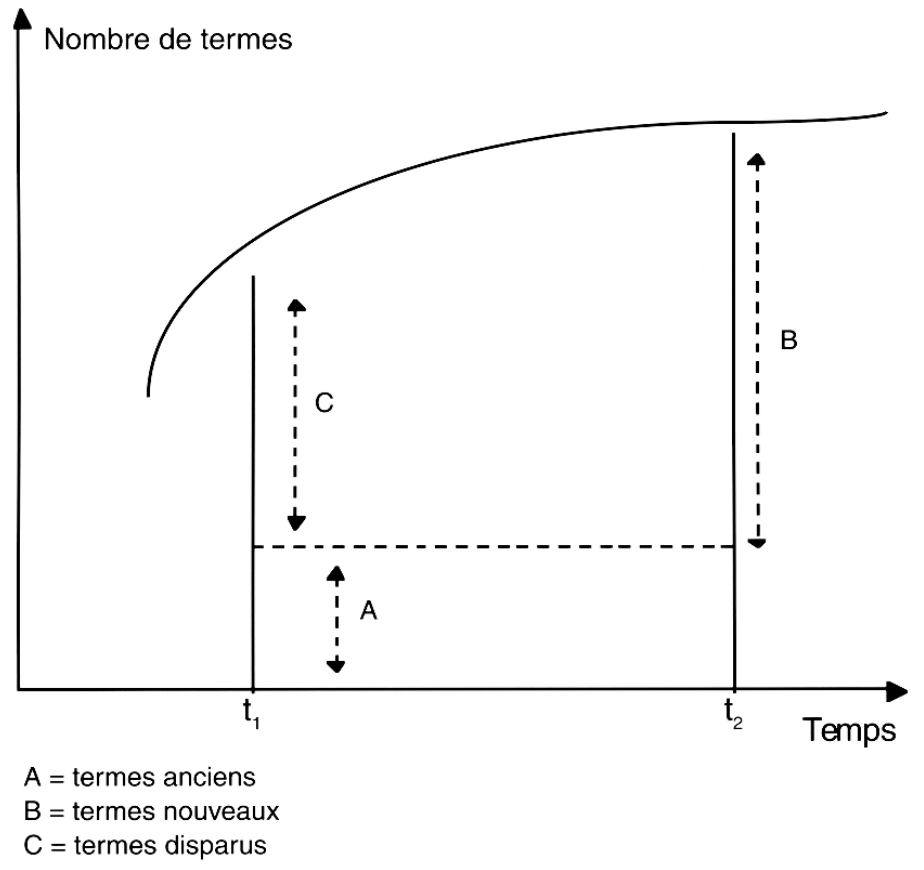

Figure 1

Nombre de terme en fonction du temps

identiques au moment $\mathrm{t} 1$ et $\mathrm{t} 2$; l'ensemble $\mathrm{B}$ renferme les signifiants qui ne se retrouvent pas à $t$, tandis que l'ensemble $\mathrm{C}$ inclut les signifiants qui ne se retrouvent pas à $t 2$. Ce sont respectivement les termes dits anciens (A), nouveaux (B) et disparus (C). Au début de l'intervalle temporel (t1) nous avons donc les ensembles $\mathrm{A}+\mathrm{C}$, à la fin de l'intervalle (t2) les ensembles A + B.

\subsection{Définitions}

Le modèle illustre l'utilité de distinguer entre croissance / décroissance et évolution.

Si la quantité des termes t 2 est supérieure à la quantité des termes $t 1$, la terminologie est dite en croissance : B > C. La notion de croissance ainsi définie traduit tout simplement le phénomène qu'est une augmentation du nombre des termes constituant une terminologie au moment $\mathrm{t} 2$ par rapport à $\mathrm{t} 1$. Inversement, la terminologie est dite en décroissance si la quantité des termes $\mathrm{t} 2$ est inférieure à la quantité des termes $\mathrm{t} 1: \mathbf{B}$ $<\mathbf{C}$.

Or, ces deux qualifications ne suffisent pas. Si le nombre des termes nouveaux et celui des termes disparus sont à peu près égaux, on aura l'impression - à tort - que rien ne bouge dans la terminologie, que celle-ci ne connait aucun changement ${ }^{7}$. Afin d'avoir une vue d'ensemble des changements, on doit considérer à la fois les quantités $\mathrm{B}$ et $\mathrm{C}$ en procédant par exemple à leur simple addition : $\mathrm{B}+\mathrm{C}$. Une notion plus approfondie traduisant le fait qu'une terminologie évolue doit donc être fonction de l'ensemble des apports et des suppressions. Elle doit également rendre compte de l'importance 
des termes qui n'ont pas changé - ceux qui ont été stables pendant le laps de temps étudié - car sans base de référence, il n'est guère possible de faire des comparaisons entre terminologies ou entre corpus. Et les notions de (dé)croissance ignorent ce qui est constant ou stable, à savoir les termes anciens.

Une définition de l'évolution pourrait être traduite par la formule $(\mathbf{B}+\mathbf{C}) / \mathbf{A}$. Le quotient traduit le rapport entre ce qui change et ce qui ne change pas. Il est supérieur à 1 s'il y a plus de changements (croissance ou décroissance) que de stabilité dans l'intervalle étudié et admet la valeur 0 s'il n'y a aucun terme nouveau ou disparu. À titre d'exemples, si dans un corpus diachronique il y a 100 termes nouveaux, 10 termes disparus et que le total des termes anciens est de 1000 , l'évolution est de $(100+10): 1000$ $=0,11$. Si un autre corpus renferme 2000 termes nouveaux, 200 termes disparus et que le total des termes anciens est de 1500 , l'évolution dans ce cas est de $(2000+200)$ : $1500=1,47$.

\subsection{Tests $^{9}$}

Après avoir présenté cette dernière formule de base visant l'aspect collectif d'une terminochronie, j'aimerais premièrement la tester sur un corpus diachronique de termes techniques en vue de connaître l'importance relative des ensembles A, B et C, et donc l'évolution de la terminologie en question. Deuxièmement, j'aimerais utiliser le modèle afin d'étudier la viabilité relative des termes des trois ensembles. Troisièmement, j'aimerais discuter d'une élaboration du modèle sous la forme d'une subdivision des ensembles dans la perspective concrète de connaître l'importance quantitative au niveau collectif du phénomène de réduction tel que défini en 2.2.

Comme terminologie technique j'ai choisi l'informatique, discipline caractérisée par une forte activité technologique, et donc terminologique, depuis quelques décennies, et où l'on doit s'attendre non seulement à un grand nombre de termes nouveaux, mais aussi à un certain nombre de termes tombés en désuétude et de termes réduits, vu la miniaturisation progressive, par exemple, et la banalisation croissante de la discipline.

Quant au corpus, j'ai choisi deux éditions d'un dictionnaire d'informatique bilingue (la première et la dernière éditions - 1970, 1985 - censé couvrir «la première ère informatique» (Pavel 1987 : 125)). Les inconvénients liés au choix d'un seul corpus diachronique du type secondaire sont très importants, certes (qualité du corpus primaire et règles de lemmatisation inconnues, manque d'information sémantique ou contextuelle, représentativité incertaine des lemmes par rapport aux termes repérés en discours, tendance conservatrice potentielle surtout quant à l'élimination des termes désuets, in casu). Mais l'avantage qu'est surtout le très haut degré de compatibilité présumé des termes-lemmes est décisif pour ce type de travail diachronique.

\subsubsection{Ensembles diachroniques et évolution}

J'ai d'abord établi le nombre des substantifs des ensembles A (termes anciens), B (termes nouveaux) et $\mathrm{C}$ (termes disparus), réparti sur les substantifs simples (1 mot graphique) et sur les substantifs complexes (2 mots graphiques ou plus). L'ensemble A comprend donc les lemmes qui se retrouvent dans les deux dictionnaires (graphies identiques).

Nous constatons (tableau 1) que le nombre des substantifs nouveaux (4 004) est un peu plus grand que celui des substantifs anciens (3 673); par contraste, l'importance des substantifs disparus (333) est limitée. Ces chiffres nous fournissent l'importance quantifiée de l'évolution, à savoir 1,18 pour le total des substantifs $((4004+33)$ : $3673)$. On note que le quotient est inférieur à 1 pour les substantifs simples 


\begin{tabular}{|l|c|c|c|}
\hline & $\begin{array}{c}\text { A } \\
\text { (termes anciens) }\end{array}$ & $\begin{array}{c}\text { B } \\
\text { (termes nouveaux) }\end{array}$ & $\begin{array}{c}\text { C } \\
\text { (termes disparus) }\end{array}$ \\
\hline Substantifs simples & 900 & 752 & 63 \\
\hline Substantifs complexes & 2773 & 3252 & 270 \\
\hline Substantifs, total & 3673 & 4004 & 333 \\
\hline
\end{tabular}

Tableau1

Nombre de substantifs

$((752+63): 900=0,91)$ mais de 1,27 pour les substantifs complexes $((3252+270)$ : $2773)$, ce qui indique que ce sont notamment ces derniers qui évoluent.

\subsubsection{Viabilité}

Deuxièmement, j'ai voulu me prononcer sur la viabilité des termes. Je suppose $a$ priori que les termes anciens (A) sont plus viables ou stables que les termes nouveaux (B) et que les termes disparus (C) en raison de leur présence à t 1 de même qu'à t2. Les termes disparus sont peut-être encore moins stables que les termes nouveaux étant donné qu'ils sont absents à t2 où les termes nouveaux engloberaient à la fois des termes «à potentiel de survie» et d'autres moins durables... Nos ensembles A, B, C peuvent-ils confirmer ces hypothèses?

La littérature (p. ex. Kocourek 1991) fait mention de certaines structures formelles traduisant la lexicalisation potentielle, ce qui refléterait la viabilité des termes : plus une structure est susceptible d'être lexicalisée, plus elle est viable. Ces indicateurs sont, entre autres, l'étendue, le nombre réduit d'articles définis, de conjonctions ou de prépositions (plus un terme est long, plus il contient d'articles définis, de conjonctions ou de prépositions, moins il y a de chances pour qu'il soit lexicalisé, donc stable ou viable). Les tableaux 2, 3, 4 et 5 montrent les résultats.

\begin{tabular}{|l|c|c|c|}
\hline & $\begin{array}{c}\text { A } \\
\text { (termes anciens) }\end{array}$ & $\begin{array}{c}\text { B } \\
\text { (termes } \\
\text { nouveaux) }\end{array}$ & $\begin{array}{c}\text { C } \\
\text { (termes disparus) }\end{array}$ \\
\hline $\begin{array}{l}\text { Longueur moyenne en } \\
\text { nombre de caractères }\end{array}$ & 20,2 & 21,1 & 23,0 \\
\hline $\begin{array}{l}\text { Longueur moyenne en } \\
\text { nombre de mots graphiques }\end{array}$ & 3,2 & 3,6 & 3,9 \\
\hline
\end{tabular}

Tableau2

Longueur moyenne des substantifs complexes

Le décompte de la longueur moyenne en nombre soit de caractères, soit de mots graphiques révèle, en effet, la hiérarchie attendue : les termes disparus sont (de deux caractères) plus longs que les termes nouveaux dont la longueur, à son tour, dépasse (de un caractère) celle des termes anciens.

Le même rang s'établit si on choisit comme étalon la présence éventuelle de préposition(s) : le pourcentage des substantifs complexes avec deux prépositions ou plus est beaucoup plus important pour les termes disparus (26,3\%) que pour les termes 


\begin{tabular}{|l|c|c|c|}
\hline & $\begin{array}{c}\text { A } \\
\text { (termes anciens) }\end{array}$ & $\begin{array}{c}\text { B } \\
\text { (termes nouveaux) }\end{array}$ & $\begin{array}{c}\text { C } \\
\text { (termes disparus) }\end{array}$ \\
\hline Deux prépositions & 10,4 & 15,8 & 21,5 \\
\hline Trois prépositions & 0,9 & 1,5 & 3,7 \\
\hline Quatre prépositions & 0,1 & 0,1 & 1,1 \\
\hline Total & 11,4 & 17,4 & 26,3 \\
\hline
\end{tabular}

Tableau3

Pourcentage de substantifs complexes avec deux prépositions ou plus

\begin{tabular}{|l|c|c|c|}
\hline & $\begin{array}{c}\text { A } \\
\text { (termes anciens) }\end{array}$ & $\begin{array}{c}\text { B } \\
\text { (termes nouveaux) }\end{array}$ & $\begin{array}{c}\text { C } \\
\text { (termes disparus) }\end{array}$ \\
\hline Articles définis & 0,04 & 0,09 & 0,11 \\
\hline Articles indéfinis & 0,02 & 0,02 & 0,02 \\
\hline Total, articles & 0,06 & 0,11 & 0,13 \\
\hline
\end{tabular}

Tableau4

Nombre d'articles par substantif complexe

\begin{tabular}{|c|c|c|}
\hline $\begin{array}{c}\text { A } \\
\text { (termes anciens) }\end{array}$ & $\begin{array}{c}\text { B } \\
\text { (termes nouveaux) }\end{array}$ & $\begin{array}{c}\text { C } \\
\text { (termes disparus) }\end{array}$ \\
\hline 28 & 19 & 0 \\
\hline
\end{tabular}

Tableau5

Nombre total de conjonctions

nouveaux $(17,4 \%)$, voire les termes anciens $(11,4 \%)$. On note que plus il y a de prépositions dans un terme, plus il est probable que ce terme appartienne à l'ensemble C (termes disparus).

La même image s'établit pour la présence éventuelle d'article(s) définis : le nombre d'articles définis est plus grand dans l'ensemble C (termes disparus) que dans B (termes nouveaux), voire A (termes anciens).

Par contre, il s'avère qu'il n'y a presque pas de conjonctions dans les lemmes nous constatons de plus qu'aucun terme disparu ne renferme de conjonction.

Bien que ce dernier test contredise les autres, il ne perturbe pas l'image globale et attendue relative à la viabilité de termes en fonction de leur structure : les termes anciens sont plus viables que les termes nouveaux qui, eux, sont plus viables que les termes disparus ${ }^{10}$.

\subsubsection{Réduction versus non-réduction}

Mon troisième but était d'étudier l'importance quantitative au niveau collectif du phénomène de réduction. Mon corpus a révélé l'importance impressionnante des termes nouveaux, mais ce sont les termes disparus qui, quoique peu nombreux, suscitent le plus grand intérêt vu leur faible viabilité et la possibilité de suivre leur destin de t1 à $\mathrm{t} 2$. 
A priori, il est à supposer qu'une partie des signifiés qui existent à t1 se retrouvent à $\mathrm{t} 2$ revêtus d'une nouvelle forme, une version réduite de la forme à $\mathrm{t} 1$. L'autre partie des signifiés $\mathrm{t} 1$ ne se retrouve pas à $\mathrm{t} 2$ dans une version réduite, soit parce que les signifiés en question sont tombés en désuétude à t2 (les nouvelles technologies évoluent vite), soit - en gros - parce que des signifiants non réduits qui y correspondent évincent par leur concurrence les formes potentielles de réduction ${ }^{11}$.

En conséquence, j'opère une subdivision de l'ensemble $C$ (termes disparus) de façon à ce qu'il soit constitué désormais de $\mathrm{C}$ réd $+\mathrm{C}$ non-réd, à savoir respectivement les signifiants qui à 2 se retrouvent en version réduite et les signifiants qui à t2 ne se retrouvent pas en version réduite (voir la figure 2).

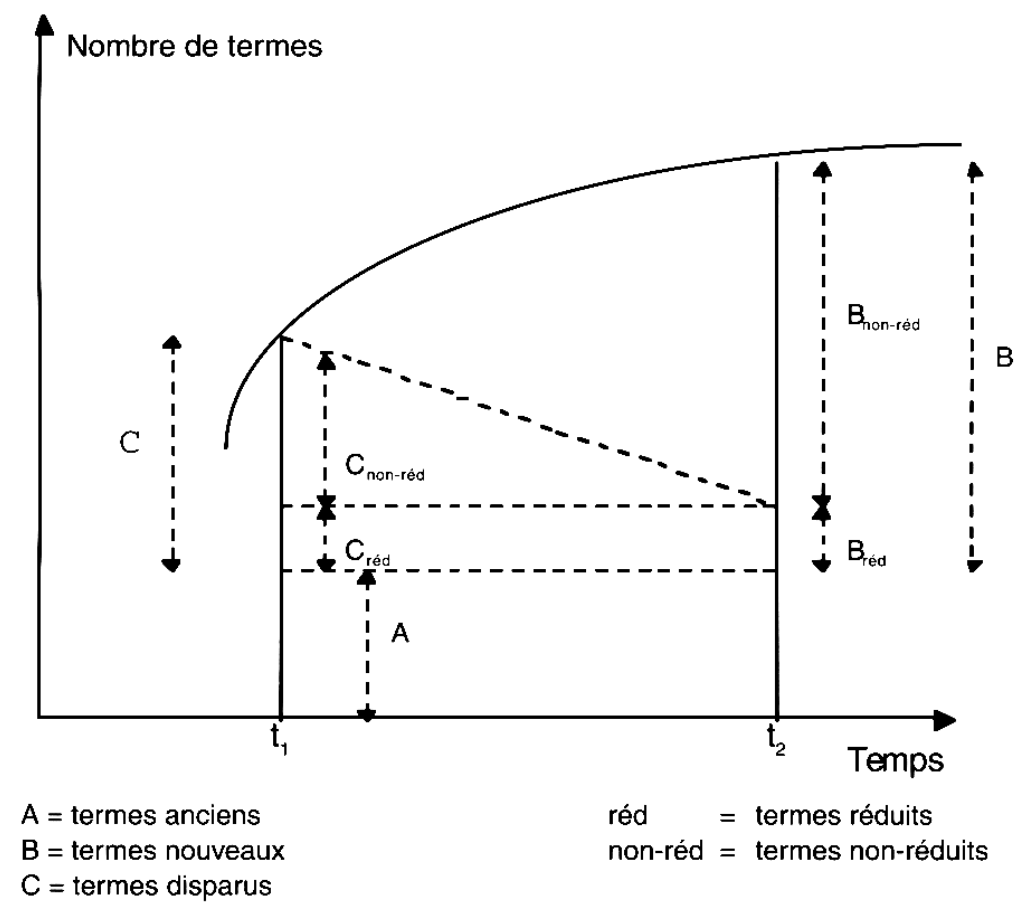

Figure 2

Nombre de termes en fonction du temps

Parallèlement, l'ensemble B sera subdivisé en deux subcatégories B réd et B nonréd. $B$ réd comprendra les signifiants qui sont des versions réduites des signifiants se trouvant dans le sous-ensemble $\mathrm{C}$ réd. $\mathrm{B}$ non-réd englobera les signifiants qui ne répondent pas à ce critère (soit parce que le signifié correspondant est nouveau, soit parce qu'il s'agit d'un synonyme non réduit qui à $\mathrm{t} 1$ faisait partie de $\mathrm{C}$ non-réd).

Comme l'ensemble $\mathrm{C}$ de mon corpus ne renferme que 270 substantifs complexes contre 3252 dans l'ensemble $\mathrm{B}$, nous voyons déjà à ce stade que l'ensemble B non-réd est nécessairement beaucoup plus important que $\mathrm{B}$ réd ${ }^{12}$. 
La réduction vue dans la perspective des signifiants nouveaux ne joue en conséquence qu'un rôle mineur. Mais, vu sous l'angle des signifiants disparus, quelle est l'importance relative de la réduction pour l'ensemble $\mathrm{C}$ ?

La réduction se manifesterait surtout comme une élision d'une suite de caractères - mots graphiques, morphèmes, fractomorphèmes - ou bien comme des signes d'intégration (voir 2.2). J'ai dû éviter, par nécessité et par simplification, toute interprétation sémantique et ai donc surtout cherché les exemples d'élision de mots de faible poids sémantique et de faible étendue, notamment les prépositions et l'article défini. Il est, en effet, bien connu qu'il y a une certaine tendance à raccourcir les mots longs ou à les créer de façon comprimée, par exemple en évitant d'interposer de entre deux substantifs (du type mémoire tampon).

Comment repérer les cas individuels de réduction présumée dans mon corpus ? Un informaticien danois a élaboré un algorithme appelé MONOMAX conçu pour traquer les fautes de frappe et les désinences de flexion. L'algorithme compare deux chaînes de caractères et établit un quotient qui traduit le nombre maximum de caractères identiques se trouvant dans le même ordre ${ }^{13}$.

Cet algorithme s'est avéré être très utile ici. Car non seulement il aurait dépisté toutes les occurrences d'élision des prépositions incolores de mes substantifs complexes, mais encore il aurait trouvé tous les exemples d'intégration du type élision des guillemets $^{14}$, lemmatisation de sigles ${ }^{15}$, ajout d'un sigle synonymique à un syntagme ${ }^{16}$.

Quels sont alors les résultats du test ${ }^{17}$ ? Il s'est avéré que 44 des 270 substantifs complexes de l'ensemble C peuvent, à l'aide de l'algorithme MONOMAX, être attribués au phénomène de réduction : 19 sont des exemples d'élision de préposition(s) et/ ou d'article ${ }^{18}, 14$ sont des cas divers d'intégration, 11 sont des exemples d'élision d'un constituant autre qu'une préposition ou un article ${ }^{19}$. Ce résultat ${ }^{20}$ peut être représenté graphiquement.

Comme l'illustre la figure 3 , nous pouvons conclure de ce dernier test que la majorité des termes «disparus» ne se retrouvera pas en version réduite à t2. À en croire le résultat de ce test de portée limitée, le phénomène de réduction ne paraît donc jouer qu'un rôle quantitativement mineur dans l'évolution d'une terminologie.

\section{PERSPECTIVES}

Avec la présente contribution, j'ai eu l'espoir d'apporter quelques précisions de base à la notion d'évolution en terminologie. J'ai également voulu esquisser nombre de considérations et d'approches au niveau du terme et au niveau de la terminologie dans son ensemble.

L'intérêt que l'on aurait à étudier le fondement de l'évolution dans ses diverses manifestations va plus loin, je crois, que l'augmentation potentielle de nos connaissances sur les caractéristiques des terminologies et donc des langues de spécialité. Outre l'aspect méthodique qui servirait à consolider la compatibilité entre différentes études qualitatives et quantitatives, la discussion systématique des modalités apparaissant par le biais d'approches différentes pourrait assurer une cohérence entre micro-niveau et macro-niveau de la terminologie, ce qui augmenterait l'utilité de cette discipline en tant que branche des sciences linguistiques.

Il est certain que beaucoup de considérations et d'analyses restent à faire dans ce domaine embryonnaire qu'est la terminochronie. Ainsi, l'expansion porte en germe au micro-niveau de même qu'au macro-niveau un potentiel qui va bien au-delà de la perspective diachronique et qui mériterait des projets de recherche finalisés. De même, il importe de continuer les réflexions sur les modalités de la réduction : le rôle de la syno- 


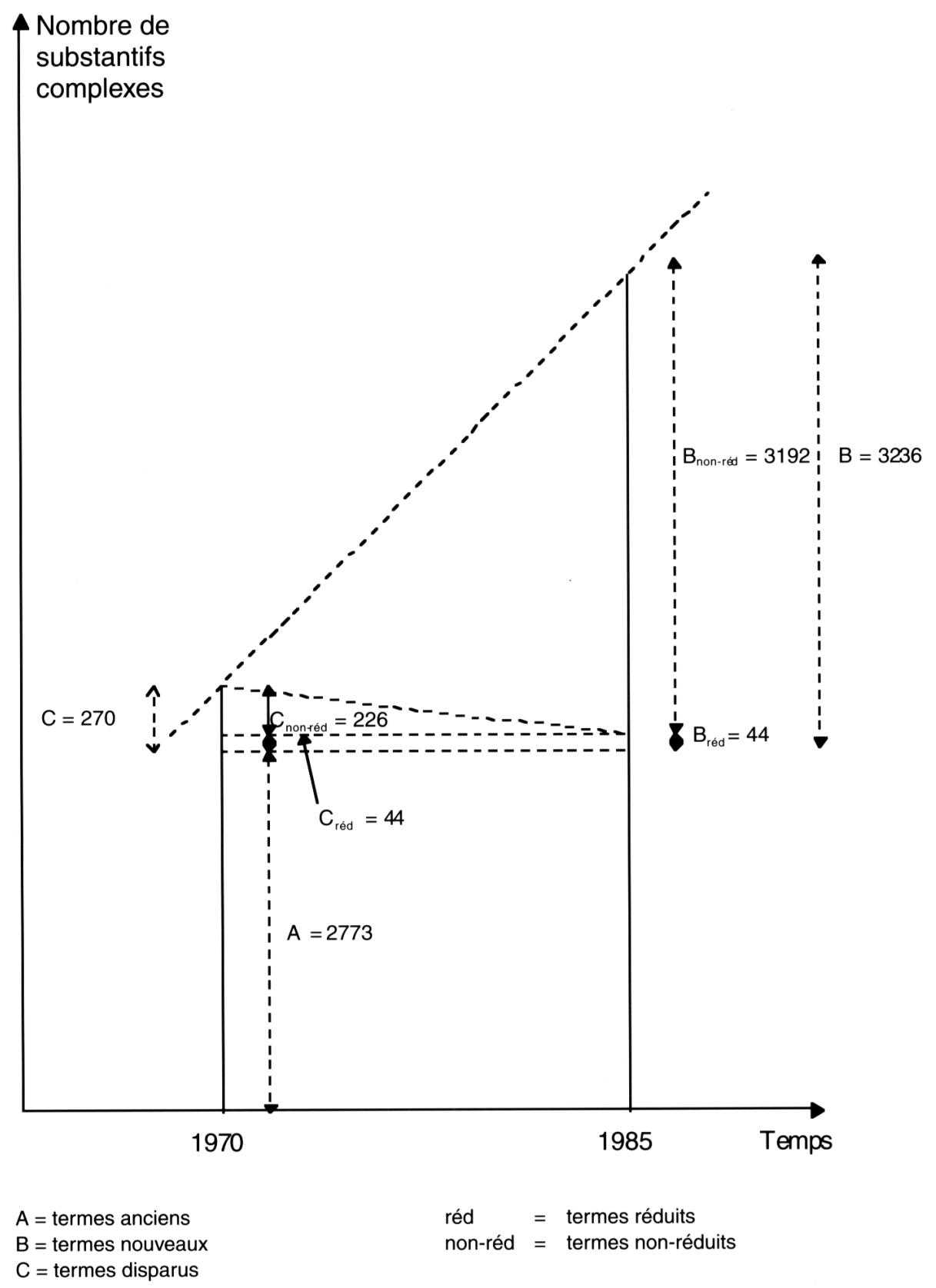

Figure 3

Nombre de substantifs complexes dans le dictionnaire Harrap's d'informatique, éditions 1970 et 1985 
nymie entre termes, de la concurrence entre matrices terminogéniques, entre chaînes de réduction... Quant à l'étude de l'évolution d'une terminologie entière, elle fait appel à l'adaptation des méthodes et outils de la linguistique informatique aux besoins spécifiques de cette branche de la terminologie.

Un plus grand nombre d'études considérant la terminologie dans une perspective diachronique augmenteraient nos connaissances sur le potentiel évolutif des structures terminologiques, ce qui intéresserait les enseignants de langues de spécialités, les traducteurs, les lexicographes, les néologues, ceux qui normalisent la terminologie et, probablement, d'autres encore.

BERNT MøLLER

École des Hautes Études Commerciales d'Aarhus, Aarhus, Danemark

\section{Notes}

1. «Cette occultation généralisée de la dimension diachronique des termes est un fait imputable à la prévalence des études synchroniques menées en linguistique au cours des vingt dernières années. Elle a été mise au profit d'abus.» (C. de Schaetzen $1993: 36$ ).

Sager (1990: 216), discutant les effets de la croissance, des changements et des innovations rapides en sciences et technologies, constate que personne ne sait dans quels domaines ou pour quels éléments se rencontrent les modifications les plus grandes réalisées pendant le laps de temps le plus petit. Aussi réclame-t-il des études pouvant nous éclairer sur l'ampleur et la vitesse de telles modifications ainsi que sur l'espérance de vie des termes.

2. «La terminologie, la terminographie et la terminotique doivent, dans un contexte d'évolution cyclique, individuelle ou collective, des désignations, définir la nature de leur objet (terme ou ensemble de termes) ainsi que les conditions de délimitation de champs terminologiques et terminographiques qui délimitent à leur tour des terminologies.» (Gouadec $1990: 26$ ).

3. Il convient peut-être ici de signaler que l'on ne doit pas s'attendre à ce que n'importe quel signifiant se réduise. La réduction est provoquée par le principe communicatif d'un maximum d'information pour un minimum d'effort. Si l'usage d'un terme se répand ou se répète, la quantité d'informations dont le récepteur a besoin pour déchiffrer son contenu - de plus en plus transparent parce que de plus en plus connu - baisse graduellement et permet ainsi un raccourcissement du terme. Or, il ne faut pas oublier que ce ne sont pas tous les termes nouveaux, loin de là, qui atteindront une audience plus large que celle des spécialistes ou qui seront fréquemment employés par ceux-ci.

4. L'établissement de telles chaînes pourrait, à titre d'exemple, apporter des précisions aux énoncés du type : «En fait, le composé lourd correspond à une première étape, c'est une formation de première génération.» (Clas $1985: 135$ ); «On voit que les formations porte-manteau et acronymiques sont déjà des créations de deuxième génération» (Clas 1985: 143); «Il s'agit [...] de la transition - graduelle, lente et évolutive - de la nature analytique des groupes de mots [GM] à la nature synthétique des mots simples [MS] [...] On peut représenter graphiquement ce phénomène de la façon suivante: GM -> MC [mots composés] -> MS» (Widlak 1991 : 191).

5. S'il y a peu de doute que la réduction opère en fonction de l'écoulement du temps, il est aussi certain qu'elle le fait en fonction d'autres facteurs comme la banalisation de l'emploi ou la normalisation.

6. Une élaboration mathématique pourrait vraisemblablement affiner le modèle.

7. Vu sous un angle néologique traditionnel, une terminologie en déclin ou un terme tombé en désuétude n'est guère intéressant, ceci peut-être parce qu'on ne prête pas attention à la disparition ou à la contraction d'un terme ou parce qu'il est nettement plus difficile de constater le non-emploi d'un terme ou d'une structure que de les attester positivement.

8. Restriction de la formule : s'il n'y a aucune constance, le modèle n'est pas valable mathématiquement.. 9. Il convient de souligner ici une faiblesse potentiellement majeure des tests, à savoir le fait que toute relation diachronique établie entre les lemmes des deux dictionnaires et entre les signifiants des ensembles A, B et $\mathrm{C}$ repose sur les présuppositions théoriquement critiquables et contradictoires 1) qu'à un signifiant ne correspond qu'un seul signifié et 2) que certaines relations de forme sont indicatrices de la constance du signifié. Cette faiblesse n'a pu être évitée étant donné le caractère du corpus.

10. Des connaissances plus approfondies en matière de dynamique des terminologies techniques apparaissent dans la passionnante étude de Ahlsved (1988). L'étude montre que la grande majorité des termes nouveaux par rapport à une période antérieure disparaît très vite : en moyenne, plus de 90 pour cent des termes nouveaux repérés au cours de périodes de dix ans (1800-1980) dans des textes allemands sur la communica- 
tion électrique des nouvelles sont même «presque» des hapax. L'étude indique aussi que la viabilité des termes constatés disparus à une époque ultérieure augmente considérablement en fonction de l'ancienneté de la première attestation des termes en question : au siècle dernier, la vie moyenne d'un terme semble être d'environ 30 ans; déjà dans les années trente, elle est inférieure à 1 an...

11. Il s'agirait dans ce dernier cas, donc, de synonymes appartenant à une autre chaîne de réduction. Ceci est une interprétation qui dépasse le cadre de l'étude, cf. note 9.

12. Je présuppose qu'à chaque signifiant $\mathrm{t} 1$ correspond au maximum un signifiant réduit à $\mathrm{t} 2$.

13. L'algorithme MOMOMAX est un quotient qui se situe dans l'intervalle $[0 ; 1]$ : «Le nombre de caractères ayant le même ordre de séquence dans les deux chaînes multiplié par deux et divisé par la somme de l'étendue des deux chaînes».

14. Ex. : test «saute mouton»-> test saute mouton.

15. Ex. : reconnaissance optique de caractères $->$ ROC [reconnaissance optique de caractères].

16. Ex. : centre de traitement de l'information $->$ centre de traitement de l'information [CTI].

17. Ont été retenues les chaînes diachroniques pour lesquelles le quotient est égal ou supérieur à 0,85 , ce qui correspond à une différence minimum de deux caractères pour une chaîne de huit caractères, au minimum. Ce choix traduit d'un côté la nécessité de repérer la quasi-totalité des cas de réduction (le quotient équivaut, par exemple, à l'élision / l'ajout de $d e$ ) et, de l'autre, le désir d'éviter trop de bruit dans les données de sortie, cf. Møller (1994).

18. Ex. : caractère de fin de support-> caractère fin de support.

19. Ex. : voyant lumineux $->$ voyant (lumineux).

20. Il convient de préciser que le nombre de «contre-exemples» (exemples d'ajout, et non d'élision d'éléments) est insignifiant.

\section{RÉFÉRENCES}

AHLSVED, Silja (1988) : «Die Entwicklung der Sprache des elektrischen Nachrichtenverkehrs als Parallele zur technischen Entwicklung», Der Ginkgobaum - Germanistisches Jahrbuch für Nordeuropa, pp. 37-42.

CAMILle, C. \& M. DEHAINE (1970) : Dictionary of Data Processing : Dictionnaire de l'informatique, Vol. II : Français-Anglais, London, Harrap.

CAMILlE, C. \& M. DEHAINE (1985) : Harrap's French and English Data Processing Dictionary, London, Paris, Harrap.

CLAS, André (1985) : «Composés lourds et créations brachygraphiques terminologiques», La Banque des mots, 30, pp. 135-145.

GOFFIN, Roger (1989) : «La terminologie des sciences et des techniques nucléaires. Un cas de diachronie récente», Terminologie diachronique, Actes du colloque organisé à Bruxelles les 25 et 26 mars 1988, Bruxelles, pp. 94-107.

GOUADEC, Daniel (1990) : Terminologie, Paris, AFNOR.

KOCOUREK, Rostislav (1991) : La langue française de la technique et de la science. Vers une linguistique de la langue savante, deuxième édition augmentée, refondue et mise à jour avec une nouvelle bibliographie, Wiesbaden, Brandstetter.

MØLLER, Bernt (1994) : «Terminologi i bevægelse», Skriften På Skærmen, Afdeling for Datalingvistik, Handelshøjskolen i Arhus», pp. 97-108.

PAVEL, Sylvia (1987) : «La terminologie de l'avenir : un dialogue Homo Sentiens-Machina Sapiens», Meta, 32 (2), pp. 124-129.

PAVEL, Sylvia (1989) : «Niveaux linguistiques et terminologie de l'intelligence artificielle», Meta, 34 (3), pp. 344-351.

PORTELANCE, Christine (1986) : «À propos du rôle des matrices terminogéniques dans le développement des langues de spécialités», Terminogramme, 39-40, pp. 1-6.

PORTELANCE, Christine (1989) : «Syntagmes et paradigmes», Meta, 34 (3), pp. 398-404.

SAGER, Juan C. (1990) : A Practical Course in Terminology Processing, Amsterdam/Philadelphia, John Benjamins.

SCHAETZEN, C. de (1993) : «Diachronie et libéralisme en terminologie», La Banque des mots, 45, pp. 3339.

WIDLAK, Stanislaw (1991): «De l'analytique au synthétique: Les mots composés en italien. Quelques remarques», Harro Stammerjohann (dir.), Analyse et synthèse dans les langues romanes et slaves, Tübingen, Gunter Narr, pp. 185-192. 